\title{
Higher Order Sobolev-Type Spaces on the Real Line
}

\author{
Bogdan Bojarski, ${ }^{1}$ Juha Kinnunen, ${ }^{2}$ and Thomas Zürcher ${ }^{3,4}$ \\ ${ }^{1}$ Institute of Mathematics, Polish Academy of Sciences, 00-956 Warsaw, Poland \\ ${ }^{2}$ Department of Mathematics, School of Science and Technology, Aalto University, P.O. Box 11100, 00076 Aalto, Finland \\ ${ }^{3}$ Department of Mathematics and Statistics, University of Jyväskylä, P.O. Box 35 (MaD), 40014 Jyväskylä, Finland \\ ${ }^{4}$ Mathematics Institute, University of Warwick, Coventry CV4 7AL, UK
}

Correspondence should be addressed to Juha Kinnunen; juha.k.kinnunen@aalto.fi

Received 16 July 2014; Accepted 6 October 2014; Published 25 November 2014

Academic Editor: Dachun Yang

Copyright ( 2014 Bogdan Bojarski et al. This is an open access article distributed under the Creative Commons Attribution License, which permits unrestricted use, distribution, and reproduction in any medium, provided the original work is properly cited.

This paper gives a characterization of Sobolev functions on the real line by means of pointwise inequalities involving finite differences. This is also shown to apply to more general Orlicz-Sobolev, Lorentz-Sobolev, and Lorentz-Karamata-Sobolev spaces.

\section{Introduction}

The general opinion in the literature on Sobolev spaces $W^{m, p}\left(\mathbb{R}^{n}\right)$ and their generalizations is that the onedimensional case $n=1$ may not be interesting. In the first order case $m=1$, the theory is essentially contained in the fundamental theorem of calculus

$$
f(x)-f(y)=\int_{x}^{y} f^{\prime}(t) d t, \quad x, y \in \mathbb{R},
$$

and the higher order case $m>1$ reduces recursively to the first order case according to the following definition.

Definition 1 (Sobolev spaces). Assume that $\Omega \subset \mathbb{R}$ is an open interval with finite Lebesgue measure in $\mathbb{R}$ and let $X$ be a vector space of functions. One says that $f \in W_{X}^{m}$ if there exists $F \in X$ that agrees with $f$ almost everywhere and has weak derivatives of order $m$ in $X$ and the derivatives of order $r, 0 \leq r \leq m-1$, are absolutely continuous.

The main goal in this paper is to point out that there are interesting phenomena related to the Sobolev spaces already in the one-dimensional case. More precisely, we give a direct and relatively elementary proof of the following result.

Theorem 2. Suppose that $\Omega \subset \mathbb{R}$ is an open interval with finite measure. A real valued (continuous) function $f: \Omega \rightarrow \mathbb{R}$ is in the Sobolev space $W^{m, p}(\Omega):=W_{L^{p}}^{m}(\Omega)(m \geq 1, p>1)$ if and only if for each $x$ and $y \in \mathbb{R}$ the mth difference of the function $f$ at the point $x$ with step $h=(y-x) / m$

$$
\Delta^{m} f(x ; y):=\Delta_{h}^{m} f(x):=\sum_{j=0}^{m}(-1)^{m-j}\left(\begin{array}{c}
m \\
j
\end{array}\right) f(x+j h)
$$

satisfies the inequality

$$
\left|\Delta^{m} f(x ; y)\right| \leq|x-y|^{m}\left[a_{f}(x)+a_{f}(y)\right]
$$

for some function $a_{f} \in L^{p}(\Omega)$.

Note that this result is false for domains with infinite measure as nonzero constant functions satisfy (3) with $a_{f}=0$ but are not in $L^{p}(\Omega)$ (we leave it to the reader to formulate an appropriate version of Theorem 2 for homogeneous Sobolev spaces, which avoids this deficiency). We will also consider this result in the more general framework of Banach function spaces. However, the material is presented in such a manner that it is accessible to readers who are only interested in the classical case. Inequality (3) in Theorem 2 is one of the examples of pointwise inequalities characterising Sobolev functions, proved and propagated by Bojarski and his students in a series of papers over the last almost 30 years; see, for example, [1-6]. The case $m=1$ lies at the initial concepts and cornerstones of analysis on general measure metric spaces [7]. 
Let us give an overview of the paper. The proof of Theorem 2 is presented in a series of results. We first give a detailed proof of the fact that Sobolev functions satisfy the pointwise inequality (3). The idea is to consider the Taylor expansion of the function. Using the linearity of the finite differences, we only need to compute and bound the finite differences of polynomials $(x-y)^{m-1}$ and of terms like $(x-y)_{+}^{m-1}$. The so-called Hermite-Genocchi formula is an integral representation of the finite differences (actually divided differences), which makes the computation rather easy. It turns out that the functions $a_{f}$ are multiples of the Hardy-Littlewood maximal function of the corresponding derivatives. Reference [8] studies the possibility to find $a_{f}$ whose definition does not involve derivatives at all. The other direction is easily shown to hold for smooth functions and the general result follows from this. Thereafter, we recall the theorems from literature necessary to be able to deduce that our main results also apply to Orlicz, Lorentz, and LorentzKaramata spaces.

\section{Sobolev Functions Satisfy Pointwise Inequality}

We refer the reader to Chapter 7 in [9] for the definition and properties of absolutely continuous functions. We write $f \in$ $L_{1}^{m}[a, b]$ to express that the function $f:[a, b] \rightarrow \mathbb{R}$ is $(m-1)$ times differentiable and that the derivative of order $m-1$ is absolutely continuous. The aim of this section is to show that membership in a Sobolev space implies (3), where the finite difference is replaced by divided differences.

Definition 3 (divided difference). Let $y_{1}, \ldots, y_{r+1}$ be points in a domain $\Omega \subset \mathbb{R}$. One assumes that if $y_{i}$ has multiplicity $n$, then $f: \Omega \rightarrow \mathbb{R}$ is $(n-1)$-times differentiable in $y_{i}$. First one considers the case where $y_{1} \leq y_{2} \leq \cdots \leq y_{r+1}$ are ordered. One defines for $r \geq 1$

$$
\begin{gathered}
{\left[y_{1}\right] f:=f\left(y_{1}\right),} \\
{\left[y_{1}, \ldots, y_{r+1}\right] f} \\
:= \begin{cases}\frac{\left[y_{2}, \ldots, y_{r+1}\right] f-\left[y_{1}, \ldots, y_{r}\right] f}{y_{r+1}-y_{1}}, & \text { if } y_{1} \neq y_{r+1}, \\
\frac{f^{(r)}\left(y_{1}\right)}{r !} & \text { otherwise. }\end{cases}
\end{gathered}
$$

We extend the definition to unordered tuples in the only possible way that makes the divided difference independent of the ordering.

Remark 4. In the recursion formula, we omitted $y_{1}$ in the nominator of the minuend and $y_{r+1}$ in the nominator of the subtrahend and then divided by $y_{r+1}-y_{r}$. We claim that we obtain the same result if we replace $y_{1}$ by $y_{k}$ and $y_{r+1}$ by $y_{m}$ as long as $y_{k} \neq y_{m}$. This can be seen by induction. We look here only at one of the two more difficult cases when one of the omitted points is either the largest or the smallest (and there are at least three points). The omitted points are denoted by a hat. We also omit the function. Consider

$$
\begin{aligned}
& \frac{\left[\hat{y}_{1}\right]-\left[\hat{y}_{m}\right]}{y_{m}-y_{1}} \\
& =\left(\frac{\left[\hat{y}_{1}, \hat{y}_{m}\right]-\left[\hat{y}_{1}, \hat{y}_{r+1}\right]}{y_{r+1}-y_{m}}-\frac{\left[\hat{y}_{1}, \hat{y}_{m}\right]-\left[\hat{y}_{m}, \hat{y}_{r+1}\right]}{y_{r+1}-y_{1}}\right) \\
& \times\left(y_{m}-y_{1}\right)^{-1} \\
& =\left(\left(y_{r+1}-y_{1}\right)\left[\hat{y}_{1}, \hat{y}_{m}\right]-\left(y_{r+1}-y_{1}\right)\left[\hat{y}_{1}, \widehat{y}_{r+1}\right]\right. \\
& \left.-\left(y_{r+1}-y_{m}\right)\left[\hat{y}_{1}, \hat{y}_{m}\right]+\left(y_{r+1}-y_{m}\right)\left[\hat{y}_{m}, \hat{y}_{r+1}\right]\right) \\
& \times\left(\left(y_{r+1}-y_{m}\right)\left(y_{m}-y_{1}\right)\left(y_{r+1}-y_{1}\right)\right)^{-1} .
\end{aligned}
$$

We collect the first and third term and expand the second one:

$$
\begin{aligned}
& \frac{\left[\widehat{y}_{1}\right]-\left[\hat{y}_{m}\right]}{y_{m}-y_{1}} \\
& =\left(\left(y_{m}-y_{1}\right)\left[\widehat{y}_{1}, \hat{y}_{m}\right]-\left(y_{m}-y_{1}\right)\left[\widehat{y}_{1}, \hat{y}_{r+1}\right]\right. \\
& \left.\quad-\left(y_{r+1}-y_{m}\right)\left[\hat{y}_{1}, \hat{y}_{r+1}\right]+\left(y_{r+1}-y_{m}\right)\left[\hat{y}_{m}, \hat{y}_{r+1}\right]\right) \\
& \quad \times\left(\left(y_{r+1}-y_{m}\right)\left(y_{m}-y_{1}\right)\left(y_{r+1}-y_{1}\right)\right)^{-1} .
\end{aligned}
$$

Collecting the first two terms and the second two, cancelling, and applying the induction hypothesis give

$$
\frac{\left[\hat{y}_{1}\right]-\left[\hat{y}_{m}\right]}{y_{m}-y_{1}}=\frac{\left[\hat{y}_{1}\right]}{y_{r+1}-y_{1}}-\frac{\left[\hat{y}_{r+1}\right]}{y_{r+1}-y_{1}}=\left[y_{1}, \ldots, y_{r+1}\right] \text {. }
$$

The following formula is a useful tool to compute divided differences.

Lemma 5 (Hermite-Genocchi formula). Let $y_{1}, \ldots, y_{r+1}$ be points in a domain $\Omega \subset \mathbb{R}$. One supposes that $f: \Omega \rightarrow \mathbb{R}$ is such that $f^{(n-1)}$ is absolutely continuous if not all $y_{i}$ coincide and that additionally $f^{(n)}\left(y_{1}\right)$ exists if all points are the same. Then

$$
\begin{aligned}
& {\left[y_{0}, \ldots, y_{n}\right] f} \\
& =\int_{0}^{1} d t_{1} \int_{0}^{t_{1}} d t_{2} \\
& \quad \ldots \int_{0}^{t_{n-1}} f^{(n)}\left(y_{0}+\left(y_{1}-y_{0}\right) t_{1}\right. \\
& \left.\quad+\cdots+\left(y_{n}-y_{n-1}\right) t_{n}\right) d t_{n} .
\end{aligned}
$$

Proof (see page 122 in [10] as well). If $n=1$, we have that

$$
\begin{aligned}
\int_{0}^{1} f^{\prime}\left(y_{0}+\left(y_{1}-y_{0}\right) t_{1}\right) d t_{1} \\
= \begin{cases}\frac{f\left(y_{1}\right)-f\left(y_{0}\right)}{y_{1}-y_{0}}, & y_{0} \neq y_{1}, \\
f^{\prime}\left(y_{0}\right), & y_{0}=y_{1},\end{cases} \\
= \begin{cases}{\left[y_{0}, y_{1}\right] f=\left[y_{1}, y_{0}\right] f,} & y_{0} \neq y_{1}, \\
{\left[y_{0}, y_{0}\right] f,} & y_{0}=y_{1} .\end{cases}
\end{aligned}
$$


Assume now that the claim is true for some $n$. We assume here that $y_{n+1} \neq y_{n}$ and leave the case when $y_{n}$ and $y_{n+1}$ coincide to the reader (computation (14) might be helpful). Then, evaluating the right most integral,

$$
\begin{aligned}
& \int_{0}^{1} d t_{1} \int_{0}^{t_{1}} d t_{2} \\
& \cdots \int_{0}^{t_{n}} f^{(n+1)}\left(y_{0}+\sum_{k=1}^{n}\left(y_{k}-y_{k-1}\right) t_{k}\right. \\
& \left.+\left(y_{n+1}-y_{n}\right) t_{n+1}\right) d t_{n+1} \\
& =\int_{0}^{1} d t_{1} \int_{0}^{t_{1}} d t_{2} \\
& \cdots \int_{0}^{t_{n-1}}\left(f ^ { ( n ) } \left(y_{0}+\sum_{k=1}^{n-1}\left(y_{k}-y_{k-1}\right) t_{k}\right.\right. \\
& \left.+\left(y_{n+1}-y_{n-1}\right) t_{n}\right) \\
& \left.-f^{(n)}\left(y_{0}+\sum_{k=1}^{n}\left(y_{k}-y_{k-1}\right) t_{k}\right)\right) d t_{n+1} \\
& \frac{1}{y_{n+1}-y_{n}} \\
& =\frac{1}{y_{n+1}-y_{n}}\left(\left[y_{0}, \ldots, y_{n-1}, y_{n+1}\right] f-\left[y_{0}, \ldots, y_{n}\right] f\right) \\
& =\left[y_{0}, \ldots, y_{n+1}\right] f \text {. }
\end{aligned}
$$

We use the Hermite-Genocchi formula to compute finite differences of polynomials.

Lemma 6. Let a be a point in $\mathbb{R}$ and $m \in \mathbb{N}_{0}$. We set

$$
p_{m, a}(y):=(y-a)^{m} \text {. }
$$

Then

$$
\left[y_{0}, \ldots, y_{n}\right] p_{m, a}= \begin{cases}0, & 0 \leq m<n \\ 1, & m=n\end{cases}
$$

Proof. As $p_{m, a}$ is smooth, we can use the Hermite-Genocchi formula, Lemma 5. If $0 \leq m<n$, then $p_{m, a}^{(n)}=0$; hence the Hermite-Genocchi formula gives the claim. If $m=n$, then $p_{m, a}^{(m)}=m$ !; we compute the integral in the Hermite-Genocchi formula via induction over $m$.

We let first for fixed $t_{0} \geq 0$

$$
\left\langle y_{0}, \ldots, y_{m}\right\rangle_{t_{0}}:=\int_{0}^{t_{0}} d t_{1} \int_{0}^{t_{1}} d t_{2} \cdots \int_{0}^{t_{m-1}} 1 d t_{m}
$$

We claim that

$$
\left\langle y_{0}, \ldots, y_{m}\right\rangle_{t_{0}}=\frac{t_{0}^{m}}{m !}, \quad m \in \mathbb{N}
$$

For $m=1$, this can easily be seen. Assume that the claim holds for some $m$. Then

$$
\begin{aligned}
\left\langle y_{0}, \ldots, y_{m+1}\right\rangle_{t_{0}} & =\int_{0}^{t_{0}} d t_{1}\left\langle y_{0}, \ldots, y_{m}\right\rangle_{t_{1}} \\
& =\int_{0}^{t_{0}} \frac{t_{1}^{m}}{m !} d t_{1}=\frac{t_{0}^{m+1}}{(m+1) !} .
\end{aligned}
$$

The Hermite-Genocchi formula tells us $\left[y_{0}, \ldots, y_{m}\right]_{p_{m, a}}=$ $\left\langle y_{0}, \ldots, y_{m}\right\rangle_{1} \cdot m$ !, giving the proof.

The following notation is on page 15 in [11].

Definition 7. Set

$$
(x-y)_{+}^{0}= \begin{cases}1, & x \geq y \\ 0, & x<y\end{cases}
$$

and for $m>1$

$$
(x-y)_{+}^{m-1}= \begin{cases}(x-y)^{m-1}, & x \geq y \\ 0, & x<y .\end{cases}
$$

The definition below is a variant of Definition 4.12 in [11]. However, as our starting point is different than Schumaker's, there is a slight regularity issue when $x$ does not differ from at least two $y_{i}$. However, we are only interested in cases where $Q_{i}^{m}$ lives under an integral, and we agree here to set $Q_{i}^{m}(x)=0$ in points where we do not have enough regularity.

Definition 8 (B-spline). Let $\cdots \leq y_{-1} \leq y_{0} \leq y_{1} \leq y_{2} \leq \cdots$ be a sequence of real numbers. Given integers $i$ and $m>0$, one defines (note that $x$ is fixed so that the finite difference is with respect to $y$ )

$$
Q_{i}^{m}(x)= \begin{cases}(-1)^{m}\left[y_{i}, \ldots, y_{i+m}\right](x-y)_{+}^{m-1}, & \text { if } y_{i}<y_{i+m} \\ 0 & \text { otherwise. }\end{cases}
$$

We call $Q_{i}^{m}$ the $m$ th order B-spline associated with the knots $y_{i}, \ldots, y_{i+m}$.

This is Theorem 2.2 in [11]. Schumaker does not give the proof but writes that it is similar to the one of Theorem 2.1 in his book.

Theorem 9 (Dual Taylor Expansion). Let $f \in L_{1}^{m}[a, b]$. Then for all $a \leq y \leq b$

$$
\begin{aligned}
f(y)= & \sum_{j=0}^{m-1} \frac{(-1)^{j} D^{j} f(b)(b-y)^{j}}{j !} \\
& +\int_{a}^{b} \frac{(x-y)_{+}^{m-1}(-1)^{m} D^{m} f(x)}{(m-1) !} d x .
\end{aligned}
$$


Proof. The proof uses induction and integration by parts. Note that, by replacing $a$ by $y$ in the integral, we may delete the + in $(x-y)_{+}$. The case $m=1$ reduces to the fundamental theorem of calculus for absolutely continuous functions; see, for example, Theorem 7.1.15 in [9]. Let us assume that the statement holds for some $m$ and fix $f \in L_{1}^{m+1}[a, b]$. Then

$$
\begin{aligned}
\int_{y}^{b} & \frac{(x-y)^{m}(-1)^{m+1} D^{m+1} f(x)}{m !} d x \\
= & \frac{(b-y)^{m}(-1)^{m+1} D^{m} f(b)}{m !} \\
& -\int_{y}^{b} \frac{(x-y)^{m-1}(-1)^{m+1} D^{m} f(x)}{(m-1) !} d x \\
= & -\frac{(b-y)^{m}(-1)^{m} D^{m} f(b)}{m !} \\
& -\sum_{j=0}^{m-1} \frac{(-1)^{j} D^{j} f(b)(b-y)^{j}}{j !}+f(y) .
\end{aligned}
$$

We now cite a part of Theorem 4.23 in [11]. Note that $m$ is a positive integer and $D_{+}^{j}$ means that we take the right hand derivative of order $j$. We only need and prove the equality for $j=0$. We also assume that not all points coincide.

Theorem 10 (Peano representation). Fix $0 \leq j \leq m-n$, where $n$ is the maximum multiplicity of $y_{i}, \ldots, y_{i+m}$. Then

$$
\left[y_{i}, \ldots, y_{i+m}\right] f=\int_{y_{i}}^{y_{i+m}} \frac{(-1)^{j} D_{+}^{j} Q_{i}^{m}(x) D^{m-j} f(x)}{(m-1) !} d x
$$

for all $f \in L_{1}^{m-j}\left[\min _{i}\left\{y_{i}\right\}, \max _{i}\left\{y_{i}\right\}\right]$.

Proof of the Peano Representation for $j=0$. We take the dual Taylor expansion from Theorem 9. Then, we apply $\left[y_{i}, \ldots, y_{i+m}\right]$ on both sides and remember that we computed the divided differences of polynomials in Lemma 6.

Our goal is to use the Peano representation to obtain an upper bound for the divided differences. We start with establishing an upper bound of the factor $D_{+} Q_{i}^{m}$ in the integrand.

Lemma 11. Suppose that $y_{i} \leq \cdots \leq y_{i+m}$ and $y_{i}<y_{i+m}$. Then there is a constant $C$ such that

$$
\left|D_{+} Q_{i}^{m}(x)\right|=\left|\left[y_{i}, \ldots, y_{i+m}\right](x-y)_{+}^{m-1}\right| \leq \frac{C}{y_{i+m}-y_{i}} .
$$

Proof. We want to estimate

$$
\begin{aligned}
& {\left[y_{i}, \ldots, y_{i+m}\right](x-y)_{+}^{m-1}} \\
& =\frac{\left[y_{i+1}, \ldots, y_{i+m}\right](x-y)_{+}^{m-1}-\left[y_{i}, \ldots, y_{i+m-1}\right](x-y)_{+}^{m-1}}{y_{i+m}-y_{i}} .
\end{aligned}
$$

Assume $k<m-1$. We claim that

$$
\frac{d^{k}}{d y^{k}}(x-y)_{+}^{m-1}=(-1)^{k}(m-1) \cdots(m-k)(x-y)_{+}^{m-1-k} \text {. }
$$

This claim is easily seen to be true for $y \neq x$. That it is true also for $y=x$ follows by induction. We also note that

$$
\frac{d^{m-2}}{d y^{m-2}}(x-y)_{+}^{m-1}
$$

is absolutely continuous on bounded intervals and that

$$
(-1)^{(m-1)}(m-1) !(x-y)_{+}^{0}
$$

is a weak derivative that equals the derivative whenever $y \neq x$. We first assume that not all $y_{j}, \ldots, y_{j+m-1}$ coincide. An application of the Hermite-Genocchi formula, Lemma 5, gives

$$
\begin{aligned}
& {\left[y_{j}, \ldots, y_{j+m-1}\right](x-y)_{+}^{m-1}} \\
& =\int_{0}^{1} d t_{1} \int_{0}^{t_{1}} d t_{2} \\
& \quad \ldots \int_{0}^{t_{m-2}}(-1)^{(m-1)}(m-1) ! \\
& \quad \cdot\left(x-\left(y_{j}+\sum_{k=1}^{m-1}\left(y_{j+k}-y_{j+k-1}\right) t_{k}\right)\right)_{+}^{0} d t_{m-1} .
\end{aligned}
$$

We use now the fact that the integrand is bounded from above by $(m-1)$ ! and the computation (14) to obtain

$$
\left|\left[y_{j}, \ldots, y_{j+m-1}\right](x-y)_{+}^{m-1}\right| \leq 1 \text {. }
$$

Suppose now that $y_{j}=\cdots=y_{j+m-1} \neq x$. Then $\left|\left[x_{j}, \ldots, x_{j+m-1}\right](x-y)_{+}^{m-1}\right|$ is bounded from above by 1 if $x>y_{i}$ and vanishes if $y_{i}<x$. In the end, we find a constant $C$ such that

$$
\left|D_{+}\left[y_{i}, \ldots, y_{i+m}\right](x-y)_{+}^{m-1}\right| \leq \frac{C}{y_{i+m}-y_{i}} .
$$

In the next result, we use the notation

$$
M g(x)=\sup _{z} f_{x}^{z}|g|
$$

for the famous Hardy-Littlewood maximal function of $g$ introduced in the seminal paper [12] of Hardy-Littlewood in Acta Math. (1930).

Theorem 12. Suppose $y_{0} \leq \cdots \leq y_{m}, y_{0}<y_{m}$ and $f$ is in $L_{1}^{m}\left[y_{0}, y_{m}\right]$ for some $m \in \mathbb{N}$. Then there is some constant $C$ such that

$$
\left|\left[y_{0}, \ldots, y_{m}\right] f\right| \leq C\left(M\left(\left|D^{m} f\right|\right)\left(y_{0}\right)+M\left(\left|D^{m} f\right|\right)\left(y_{m}\right)\right) \text {. }
$$


Proof. We use the Peano representation, Theorem 10, with $i=j=0$. Then, using the bound of $\left|D_{+} Q_{i}^{m}(x)\right|$ found in Lemma 11,

$$
\begin{aligned}
\left|\left[y_{0}, \ldots, y_{m}\right] f\right| & \leq \frac{1}{(m-1) !} \int_{y_{0}}^{y_{m}}\left|D_{+} Q_{i}^{m}(x)\right|\left|D^{m} f\right|(x) d x \\
& \leq \frac{C}{y_{m}-y_{0}} \int_{y_{0}}^{y_{m}}\left|D^{m} f\right|(x) d x .
\end{aligned}
$$

For $y \in\left[y_{0}, y_{m}\right]$, we have

$$
\begin{aligned}
& \left|\left[y_{0}, \ldots, y_{m}\right] f\right| \\
& \quad \leq \frac{C}{y_{m}-y_{0}}\left(\int_{y_{0}}^{y}\left|D^{m} f\right|(x) d x+\int_{y}^{y_{m}}\left|D^{m} f\right|(x) d x\right)
\end{aligned}
$$

and further

$$
\begin{aligned}
& \left|\left[y_{0}, \ldots, y_{m}\right] f\right| \\
& \leq C\left(f_{y_{0}}^{y} \frac{y-y_{0}}{y_{m}-y_{0}}\left|D^{m} f\right|(x) d x\right. \\
& \left.\quad+\int_{y}^{y_{m}} \frac{y_{m}-y}{y_{m}-y_{0}}\left|D^{m} f\right|(x) d x\right) .
\end{aligned}
$$

The first factors in both integrals are bounded from above by 1 . We see that the integrals are bounded from above by the corresponding maximal operators. This gives the claim.

The finite differences defined in (2) are, up to a factor, divided differences for equispaced nodes.

Lemma 13. Let $x, y$ be two points in a domain $\Omega \subset \mathbb{R}$. Let $m \in \mathbb{N}$ and $x_{k}=x_{0}+h k$, where $h=(y-x) / m$. Then each $f: \Omega \rightarrow \mathbb{R}$ satisfies

$$
\left[x_{0}, \ldots, x_{m}\right] f=\frac{1}{h^{m} m !} \Delta^{m} f(x ; y) \quad\left(x=x_{0}, x_{m}=y\right) .
$$

Proof. We use induction. For $m=1$, we have

$$
\left[x_{0}, x_{1}\right] f=\frac{f\left(x_{1}\right)-f\left(x_{0}\right)}{x_{1}-x_{0}} .
$$

As $h=x_{1}-x_{0}, x=x_{0}, y=x_{1}$, and $m=1$, we obtain the claim. Suppose that the claim is true for some $m \in \mathbb{N}$. Then

$$
\begin{array}{rl}
\Delta_{h}^{m+1} & f(x) \\
& =\sum_{j=0}^{m+1}(-1)^{m+1-j}\left(\begin{array}{c}
m+1 \\
j
\end{array}\right) f(x+j h) \\
& =\sum_{j=0}^{m+1}(-1)^{m+1-j}\left(\left(\begin{array}{c}
m \\
j-1
\end{array}\right)+\left(\begin{array}{c}
m \\
j
\end{array}\right)\right) f(x+j h)
\end{array}
$$

$$
\begin{aligned}
= & \sum_{j=0}^{m+1}(-1)^{m-j}\left(\begin{array}{c}
m \\
j
\end{array}\right) f(x+(j+1) h) \\
& -\sum_{j=0}^{m+1}(-1)^{m-j}\left(\begin{array}{c}
m \\
j
\end{array}\right) f(x+j h) \\
= & \sum_{j=0}^{m}(-1)^{m-j}\left(\begin{array}{c}
m \\
j
\end{array}\right) f\left(x_{1}+j h\right) \\
& -\sum_{j=0}^{m}(-1)^{m-j}\left(\begin{array}{c}
m \\
j
\end{array}\right) f(x+j h) \\
= & h^{m} m !\left(\left[x_{1}, \ldots, x_{m+1}\right] f-\left[x_{0}, \ldots, x_{m}\right] f\right) \\
= & h^{m} m !(h(m+1))\left[x_{0}, \ldots, x_{m+1}\right] f .
\end{aligned}
$$

We can now formulate Theorem 12 in terms of finite differences.

Corollary 14 (Corollary of Theorem 12). Let $\Omega \subset \mathbb{R}$ be a domain. Suppose $f \in L_{1}^{m}(\Omega)$ for some $m \in \mathbb{N}$; then there is a constant $C=C(m)$ such that

$$
\begin{aligned}
& \left|\Delta^{m} f(x ; y)\right| \\
& \quad \leq C|x-y|^{m}\left(M\left(\left|D^{m} f\right|\right)(x)+M\left(\left|D^{m} f\right|\right)(y)\right),
\end{aligned}
$$

$$
x, y \in \Omega .
$$

In particular, we have the following special case. Suppose $X$ and $Y$ are normed spaces of functions such that the maximal operator $M: X \rightarrow Y$ is bounded. If $f \in W_{X}^{m}(\Omega)$ for some $m \geq 1$, then

$$
\left|\Delta^{m} f(x ; y)\right| \leq|x-y|^{m}\left(a_{f}(x)+a_{f}(y)\right)
$$

for some function $a_{f} \in Y$ depending on $f$.

Proof. We first use Lemma 13 and then Theorem 12 with step $h=(y-x) / m$. There is some constant $C=C(m)$ such that

$$
\begin{aligned}
& \left|\Delta^{m} f(x ; y)\right| \\
& \quad=h^{m} m !\left|\left[x_{0}, \ldots, x_{m}\right] f\right| \\
& \quad \leq C h^{m}\left(M\left(\left|D^{m} f\right|\right)(x)+M\left(\left|D^{m} f\right|\right)(y)\right) \\
& \quad \leq|x-y|^{m}\left(C M\left(\left|D^{m} f\right|\right)(x)+C M\left(\left|D^{m} f\right|\right)(y)\right) .
\end{aligned}
$$

Proof of Theorem 2 (Sobolev functions satisfy pointwise inequality). We combine Corollary 14 with the boundedness of the maximal operator, which is by now classical and can be found, for example, in Theorem 3.3.10 in [13]. 


\section{Pointwise Inequality Implies Membership in Sobolev Space}

In this section, our aim is to show that the pointwise inequality (3) implies the existence of the highest order derivative in the correct space. We will take care of the intermediate derivatives in the next section. In this section, the properties of the Lebesgue spaces play an important role. Our point of view is however to extract the properties of the Lebesgue spaces and base our proofs solely on these properties. We hope that the disadvantage of being slightly more abstract is later on compensated when we generalize the results. We start with some auxiliary results.

Lemma 15. Let $\Omega \subset \mathbb{R}$ be a domain and $\varphi \in \mathscr{C}_{0}^{n}(\Omega)$; that is, $\varphi$ has compact support in $\Omega$ and is n-times continuously differentiable. Then

$$
\left|\left[x_{0}, \ldots, x_{n}\right] \varphi-\frac{\varphi^{(n)}(y)}{n !}\right| \longrightarrow 0
$$

uniformly in $\Omega$ as $x_{0}, \ldots, x_{n} \rightarrow y$.

Proof. By Taylor's formula, for $x \in \operatorname{conv}\left\{x_{0}, \ldots, x_{n}\right\}$, there is $\xi_{x} \in \operatorname{conv}\left\{x_{0}, \ldots, x_{n}\right\}$ such that

$$
\begin{aligned}
\varphi(x)= & \varphi(y)+\varphi^{\prime}(y)(x-y)+\cdots+\varphi^{(n-1)}(y) \frac{(x-y)^{n-1}}{(n-1) !} \\
& +\frac{1}{n !} \varphi^{(n)}\left(\xi_{x}\right)(x-y)^{n} .
\end{aligned}
$$

We apply $\left[x_{0}, \ldots, x_{n}\right]$ on both sides (with variable $x$ ) and use the computation of the divided differences of polynomials carried out in Lemma 6 to obtain

$$
\left[x_{0}, \ldots, x_{n}\right] \varphi(x)=\frac{1}{n !} \varphi^{(n)}\left(\xi_{x}\right) .
$$

As $\varphi^{(n)}$ is uniformly continuous on compact intervals, the claim follows.

Lemma 16. Assume $\Omega \subset \mathbb{R}$ is open and $K \subset \Omega$ is compact. Then there is a neighborhood of $K$ that is a subset of $\Omega$.

Proof. For each $x \in K$, we find a ball $B\left(x, r_{x}\right) \subset \Omega$. Suppose the statement of the lemma is false. Then for every $n \in \mathbb{N}$ there is $z_{n} \in \Omega \backslash K$ with $\operatorname{dist}\left(z_{n}, K\right)<1 / n$. Thus, for each $n \in \mathbb{N}$, there is $y_{n} \in K$ with $d\left(z_{n}, y_{n}\right)<1 / n$. Since $K$ is compact, there is a subsequence $\left(y_{n_{k}}\right)_{k}$ of $\left(y_{n}\right)_{n}$ that converges to some $y \in K$. Now, $y$ lies in one of the balls $B\left(x, r_{x}\right) \subset \Omega$. For $k$ large enough, we have that $y_{n_{k}}$ and $z_{n_{k}}$ are in $B\left(x, r_{x}\right) \subset \Omega$. This is a contradiction.

Lemma 17. Suppose $\Omega \subset \mathbb{R}$ is open and $K \subset \Omega$ compact. Then there exists $\varepsilon>0$ such that

$$
(\Omega-h) \cap K=\Omega \cap K
$$

for $|h|<\varepsilon$.
Proof. We first show that any member of the left hand side is contained in the right hand side; this is true for any $h \in \mathbb{R}$. If $z$ is a member of the left hand side, then it is in $K$, and since $K$ is a subset of $\Omega$, it is also a member of the right hand side. To prove the other inclusion, note that, by Lemma 16, there is $\varepsilon>0$ such that the $\varepsilon$-neighborhood $K_{\varepsilon}$ of $K$ lies in $\Omega$. Now suppose that $z$ is in the right hand side and $|h|<\varepsilon$. Then for $x:=z+h$, we have $x \in K_{\varepsilon} \subset \Omega$ implying that $z=x-h \in \Omega-h$. That $z \in K$ is clear. This gives the proof.

Next, we state basically Definition 3.1.1 in [14]. Readers following [13] might want to have a look at Definitions 1.1.1 and 1.1.3 in their book. To gain familiarity with the concept of Banach function norm, the reader may want to verify that Lebesgue spaces are examples of Banach function spaces. As we are here only interested in Lebesgue measure, we adapt the notion to this case. The set $\mathscr{M}^{+}$denotes the cone of measurable functions whose values lie in $[0, \infty]$.

Definition 18 (Banach function norm; Banach function space). Assume that $R \subset \mathbb{R}$. A Banach function norm on $R$ is a map $\rho$ from $\mathscr{M}^{+}(R)$ to $[0, \infty]$ such that, for all $f, g$, and $f_{n}(n \in \mathbb{N})$ in $\mathscr{M}^{+}(R)$, all scalars $\lambda \geq 0$, and all measurable subsets $E$ of $R$, the following are true, where $\mu$ is the Lebesgue measure:

(P1) $\rho(f)=0$ if, and only if, $f=0 \mu$-almost everywhere, $\rho(\lambda f)=\lambda \rho(f), \rho(f+g) \leq \rho(f)+\rho(g) ;$

(P2) if $0 \leq g \leq f \mu$-almost everywhere, then $\rho(g) \leq \rho(f)$;

(P3) if $0 \leq f_{n} \uparrow f \mu$-almost everywhere, we have $\rho\left(f_{n}\right) \uparrow$ $\rho(f)$

(P4) if $\mu(E)<\infty$, then $\rho\left(\chi_{E}\right)<\infty$;

(P5) if $\mu(E)<\infty$, then there is a constant $C=C(E, \rho)<\infty$ such that $\int_{E} f d x \leq C \rho(f)$.

Given such a function norm $\rho$, the set $X=X(\rho)$ of all extended measurable functions $f$ (identifying functions equal $\mu$-almost everywhere) such that $\rho(|f|)<\infty$ is called a Banach function space (BFS for short), and we define

$$
\|f\|_{X}=\rho(|f|), \quad f \in X .
$$

We leave it to the reader to verify that Lebesgue spaces satisfy the following properties $\left(X^{\prime}\right.$ can be chosen as the dual space).

Properties 19. Given a Banach function space $X$, we require the existence of a Banach function space $X^{\prime}$ such that

(B) $X$ contains each essentially bounded function whose support has finite measure,

(HI) Hölder's inequality: $\int|f g| d x \leq\|f\|_{X}\|g\|_{X^{\prime}}$,

(C) $X^{\prime}$ is complete,

(D) $\|g\|_{X}=\sup \left\{\int_{\Omega}|g| h: h \in \mathscr{M}^{+},\|h\|_{X^{\prime}} \leq 1\right\}$,

(AC) absolutely continuous norm: if $\left(\chi_{E_{n}}\right)_{n}$ is a sequence of characteristic functions converging pointwise almost everywhere to 0 , then $\left\|f \chi_{E_{n}}\right\|_{X^{\prime}}$ converges to zero for all $f \in X^{\prime}$, 
(A) each function $f \in X^{\prime}$ can be approximated by simple functions (in $X^{\prime}$-norm),

(NC) if $f \in X^{\prime}$ and $h_{n}$ are step functions with $0 \leq h_{n} \uparrow|f|$, then

$$
\left\|f-h_{n} \operatorname{sgn}(f)\right\|_{X^{\prime}} \longrightarrow 0 \quad \text { as } n \longrightarrow \infty .
$$

Lemma 20. Suppose $\Omega \subset \mathbb{R}$ is open and $X$ is a Banach function space fulfilling the items listed in Properties 19. Assume that $f: \Omega \rightarrow \mathbb{R}$ is in $X$. We let $\lambda_{f}: X^{\prime} \cap \mathscr{C}_{0}^{r+1}(\Omega) \rightarrow \mathbb{R}$ be defined as

$$
\lambda_{f}(\varphi)=\int_{\Omega} f \varphi^{(r)} d x
$$

If

$$
\begin{array}{r}
\left|\Delta^{r} f(x, y)\right| \leq|x-y|^{r}\left[a_{f}(x)+a_{f}(y)\right], \\
\text { for almost every } x, y \in \Omega
\end{array}
$$

for some $a_{f} \in X$, then $\lambda_{f}$ has a continuous extension to $X^{\prime}$ with norm bounded from above by $2 r^{r}\left\|a_{f}\right\|_{X}$.

Proof. Our goal is to show

$$
\left|\int_{\Omega} f(x) \varphi^{(r)}(x) d x\right| \leq 2 r^{r}\left\|a_{f}\right\|_{X}\|\varphi\|_{X^{\prime}},
$$

because $\lambda_{f}$ extends then by the theorem of Hahn-Banach to a bounded functional on $X^{\prime}$ with norm bounded from above by $2 r^{r}\left\|a_{f}\right\|_{X}$. Without loss of generality, we may assume that $a_{f}$ is nonnegative. If $f$ were be smooth, we could just use integration by parts and use as bound the limiting case of the pointwise inequality (48). As $f$ is not necessarily smooth, we have to operate on the level of finite differences.

Let us choose $\varphi \in \mathscr{C}_{0}(\Omega)$ and denote its support by $K$. Lemma 17 ensures the existence of some positive $\varepsilon>0$ such that $(\Omega-j h) \cap K=\Omega \cap K$ for $0 \leq j \leq r$ as long as $|h|<\varepsilon / r$. We assume in the following that $h$ has been chosen accordingly.

The following integrals exist by Hölder's inequality because $\varphi$ and thus $\Delta_{-h}^{r} \varphi(x)$ are bounded with compact support and $f$ is integrable. Consider

$$
\begin{aligned}
\int_{\Omega} & f(x) \varphi^{(r)}(x) d x \\
= & \int_{\Omega} f(x)(-h)^{-r} \Delta_{-h}^{r} \varphi(x) d x \\
& \quad+\int_{\Omega} f(x)\left(\varphi^{(r)}(x)-(-h)^{-r} \Delta_{-h}^{r} \varphi(x)\right) d x .
\end{aligned}
$$

We compute the following integral:

$$
\begin{aligned}
\int_{\Omega} & f(x) \Delta_{-h}^{r} \varphi(x) d x \\
= & \int_{\Omega} f(x) \sum_{j=0}^{r}(-1)^{r-j}\left(\begin{array}{l}
r \\
j
\end{array}\right) \varphi(x-j h) d x \\
= & \sum_{j=0}^{r}(-1)^{r-j}\left(\begin{array}{l}
r \\
j
\end{array}\right) \int_{\Omega} f(x) \varphi(x-j h) d x
\end{aligned}
$$

$$
\begin{aligned}
& =\sum_{j=0}^{r}(-1)^{r-j}\left(\begin{array}{l}
r \\
j
\end{array}\right) \int_{\Omega-j h} f(y+j h) \varphi(y) d y \\
& =\sum_{j=0}^{r}(-1)^{r-j}\left(\begin{array}{l}
r \\
j
\end{array}\right) \int_{(\Omega-j h) \cap K} f(y+j h) \varphi(y) d y .
\end{aligned}
$$

We have chosen $\varepsilon>0$ such that $(\Omega-j h) \cap K=\Omega \cap K$. We continue

$$
\begin{aligned}
\int_{\Omega} & f(x) \Delta_{-h}^{r} \varphi(x) d x \\
= & \sum_{j=0}^{r}(-1)^{r-j}\left(\begin{array}{l}
r \\
j
\end{array}\right) \int_{\Omega \cap K} f(y+j h) \varphi(y) d y \\
= & \sum_{j=0}^{r}(-1)^{r-j}\left(\begin{array}{l}
r \\
j
\end{array}\right) \int_{\Omega} f(y+j h) \varphi(y) d y \\
= & \int_{\Omega} \sum_{j=0}^{r}(-1)^{r-j}\left(\begin{array}{c}
r \\
j
\end{array}\right) f(y+j h) \varphi(y) d y \\
= & \int_{\Omega} \Delta_{h}^{r} f(x) \varphi(x) d x .
\end{aligned}
$$

With the aid of (52), we can now rewrite (50) as

$$
\begin{aligned}
\int_{\Omega} f & (x) \varphi^{(r)}(x) d x \\
= & \int_{\Omega}(-h)^{-r} \Delta_{h}^{r} f(x) \varphi(x) d x \\
& \quad+\int_{\Omega} f(x)\left(\varphi^{(r)}(x)-(-h)^{-r} \Delta_{-h}^{r} \varphi(x)\right) d x .
\end{aligned}
$$

Inequality (48) leads to

$$
\begin{aligned}
& \left|\int_{\Omega} f(x) \varphi^{(r)}(x) d x\right| \\
& \quad \leq r^{r} \int_{\Omega}\left(a_{f}(x)+a_{f}(x+r h)\right)|\varphi(x)| d x \\
& \quad+\int_{\Omega}|f(x)|\left|\varphi^{(r)}(x)-(-h)^{-r} \Delta_{-h}^{r} \varphi(x)\right| d x .
\end{aligned}
$$

Using Hölder's inequality (HI) for the first summand, we are left to show that the second summand tends to 0 as $h$ approaches 0 . We note first that since the second factor has support in a set of finite measures, we can replace the integration domain by a set of finite measures. Then we apply a $\left(L^{1}, L^{\infty}\right)$-Hölder inequality and the uniform convergence of the divided difference to $\varphi^{(r)}(x) / r$ ! established in Lemmas 13 and 15 .

After having verified that $\lambda_{f}$ has a continuous extension to $X^{\prime}$, we want to show that this extension has a representation as integral. 
Lemma 21. Assume $\Omega \subset \mathbb{R}$ is open. Suppose the properties listed in Properties 19 are met. Let $\lambda_{f}$ be defined as in (47) and bounded on $X^{\prime}$. Then there exists $g \in X$ with $\|g\|_{X} \leq\left\|\lambda_{f}\right\|$ such that

$$
\lambda_{f}(\varphi)=(-1)^{r} \int_{\Omega} g(x) \varphi(x) d x
$$

for all $\varphi \in X^{\prime}$, especially for all $\varphi \in \mathscr{C}_{0}^{\infty}(\Omega)$.

Proof. The proof is heavily influenced by the proof of Theorem 1.4.1 in [13]. We first want to find a signed measure $v$ such that

$$
\lambda_{f}(\varphi)=\int_{\Omega} \varphi d \nu
$$

for each $\varphi \in X^{\prime}$. Then, we want to apply the LebesgueRadon-Nikodym theorem to replace $d v$ by $(-1)^{r} g(x) d x$ for some integrable function $g$ for which we then show that it is contained in $X$.

We start by assuming that the measure of $\Omega$ is finite. For each $\mu$-measurable set $A$, we set

$$
\nu(A):=\lambda_{f}\left(\chi_{A}\right) .
$$

We start by proving the $\sigma$-additivity of $\nu$ (for the $\mu$ measurable sets). In the first step, we just show the additivity. By induction, it is enough to consider only two sets.

Let $A_{1}$ and $A_{2}$ be two disjoint $\mu$-measurable sets. Then

$$
\begin{aligned}
\nu\left(A_{1} \cup A_{2}\right) & =\lambda_{f}\left(\chi_{A_{1} \cup A_{2}}\right)=\lambda_{f}\left(\chi_{A_{1}}+\chi_{A_{2}}\right) \\
& =\lambda_{f}\left(\chi_{A_{1}}\right)+\lambda_{f}\left(\chi_{A_{2}}\right)=v\left(A_{1}\right)+v\left(A_{2}\right) .
\end{aligned}
$$

We move to the verification of the $\sigma$-additivity. Let $A_{1}, A_{2}, \ldots$ be countably many pairwise disjoint $\mu$-measurable sets. We first want to show that $\sum_{i} \chi_{A_{i}}$ converges in $X^{\prime}$.

We let $E_{k}:=\cup_{i=1}^{k} A_{i}$ and $E:=\cup_{i=1}^{\infty} A_{i}$. Then, since $\left(E_{k}\right)_{k}$ is increasing,

$$
\mu(E)=\mu\left(\bigcup_{k=1}^{\infty} E_{k}\right)=\lim _{k \rightarrow \infty} \mu\left(E_{k}\right) .
$$

As $E$ has finite measure, given $\varepsilon>0$, there exists $k_{0} \in \mathbb{N}$ such that

$$
(1-\varepsilon) \mu(E)<\mu\left(E_{k_{0}}\right) .
$$

By the disjointness of the sets $A_{i}$, we see that

$$
\mu(E)=\mu\left(E_{k_{0}}\right)+\mu\left(\bigcup_{i=k_{0}+1}^{\infty} A_{i}\right) .
$$

It follows that

$$
\begin{aligned}
\mu\left(\bigcup_{i=k_{0}+1}^{\infty} A_{i}\right) & =\mu(E)-\mu\left(E_{k_{0}}\right)<\mu(E)-(1-\varepsilon) \mu(E) \\
& =\varepsilon \mu(E) .
\end{aligned}
$$

We have for $m<n$

$$
\left\|\sum_{i=1}^{n} \chi_{A_{i}}-\sum_{i=1}^{m} \chi_{A_{i}}\right\|_{X^{\prime}}=\left\|\sum_{i=m+1}^{n} \chi_{A_{i}}\right\|_{X^{\prime}} \leq\left\|\chi_{\left.\cup_{i=m+1}^{\infty}\right)} A_{i}\right\|_{X^{\prime}} .
$$

By the considerations before and the fact that $\|\cdot\|_{X^{\prime}}$ is absolutely continuous as stipulated in (AC), we see that $\sum_{i=1}^{n} \chi_{A_{i}}$ is a Cauchy sequence. Now, as $X^{\prime}$ is complete by (C), the Cauchy sequence converges in $X^{\prime}$. We have, by the continuity of $\lambda_{f}$,

$$
\begin{aligned}
\nu\left(\cup_{i} A_{i}\right) & =\lambda_{f}\left(\chi_{\cup_{i} A_{i}}\right)=\lambda_{f}\left(\sum_{i=1}^{\infty} \chi_{A_{i}}\right) \\
& =\lim _{n \rightarrow \infty} \lambda_{f}\left(\sum_{i=1}^{n} \chi_{A_{i}}\right)=\lim _{n \rightarrow \infty} \sum_{i=1}^{n} \lambda_{f}\left(\chi_{A_{i}}\right) \\
& =\lim _{n \rightarrow \infty} \sum_{i=1}^{n} v\left(A_{i}\right)=\sum_{i=1}^{\infty} v\left(A_{i}\right) .
\end{aligned}
$$

Thus $v$ is $\sigma$-additive.

In order to apply the theorem of Lebesgue-RadonNikodym, see, for example, Section 6.9 in [15], and concluding the existence of $\hat{g} \in L^{1}(\Omega)$ such that

$$
\nu(A)=\int_{A} \hat{g} d x, \quad A \mu \text {-measurable, }
$$

we need to show that $v$ is absolutely continuous with respect to $\mu$. To do so, assume that $\mu(N)=0$. Then $\nu(N)=\lambda_{f}\left(\chi_{N}\right)$. By the absolute continuity of the norm of $X^{\prime}$ required in (AC), we see that $\left\|\chi_{N}\right\|_{X^{\prime}}=0$, and therefore $\nu(N)=0$. Replacing $\hat{g}$ by $-\widehat{g}$ if necessary, we obtain

$$
\lambda_{f}\left(\chi_{A}\right)=(-1)^{r} \int_{\Omega} \widehat{g}(x) \chi_{A}(x) d x .
$$

Now, we assume that $\Omega$ does not necessarily have finite measure. We write $\Omega=\cup_{n} \Omega_{n}$ as union of open sets with finite measure such that $\Omega_{n} \subset \Omega_{n+1}$. By the considerations above, we find for each $n \in \mathbb{N}$ a $g_{n}$ such that

$$
\lambda_{f}\left(\chi_{A}\right)=(-1)^{r} \int_{\Omega} g_{n}(x) \chi_{A}(x) d x
$$

for each measurable set $A \subset \Omega_{n}$. It is easily seen that $g_{n}$ and $g_{n+1}$ agree almost everywhere on $\Omega_{n}$. This gives rise to a function $g$ satisfying

$$
\begin{array}{r}
\lambda_{f}\left(\chi_{A}\right)=(-1)^{r} \int_{\Omega} g(x) \chi_{A}(x) d x \\
A \subset \bigcup_{n=1}^{N} \Omega_{n} \text { for some } N .
\end{array}
$$

By linearity, we have for each simple function $s$ :

$$
\lambda_{f}(s)=(-1)^{r} \int_{\Omega} g(x) s(x) d x .
$$

To show that $g \in X$, we want to use property (D). 
Let $h$ be a nonnegative simple function with support in some $\Omega_{n}$. Then $h \operatorname{sgn}(g)$ is also a simple function with support in $\Omega_{n}$. It is a finite linear combination of characteristic functions of sets in $\Omega_{n}$. Hence

$$
\int|h g| d x=\int h \cdot \operatorname{sgn}(g) g d x=\lambda_{f}(h \operatorname{sgn}(g)) \text {. }
$$

Using the boundedness of $\lambda_{f}$, we see that

$$
\int|h g| d x \leq\left\|\lambda_{f}\right\|\|h\|_{X^{\prime}}
$$

If $h$ is an arbitrary function in $X^{\prime}$, then we may construct a sequence $\left(h_{n}\right)_{n=1}^{\infty}$ of simple functions, each $h_{n}$ having support in $\Omega_{n}$, such that $0 \leq h_{n} \uparrow|h| \mu$-almost everywhere. By the monotone convergence theorem, we have by (71) that

$$
\int|h g| d x=\lim _{n \rightarrow \infty} \int\left|h_{n} g\right| d x \leq \limsup _{n \rightarrow \infty}\left\|\lambda_{f}\right\|\left\|h_{n}\right\|_{X^{\prime}}
$$

By $(\mathrm{P} 3)$ in Definition 18 , we see that

$$
\int|h g| d x \leq\left\|\lambda_{f}\right\|\|h\|_{X^{\prime}}
$$

and Property (D) gives that $g \in X$. In the last step, we want to upgrade the integral representation (69) to hold for arbitrary functions in $\varphi \in X^{\prime}$. As before, we approximate $\varphi$ by simple functions $h_{n}$ with support contained in $\Omega_{n}$ and $0 \leq h_{n} \uparrow|\varphi|$ in $\mu$-almost everywhere point. Define $s_{n}:=h_{n} \operatorname{sgn}(\varphi)$. By (NC), $s_{n}$ converges to $\varphi$ in $X^{\prime}$ and as both $\lambda_{f}$ and "its integral representation" are continuous and agree on the set of step functions, they agree on $X^{\prime}$.

Having shown the existence of the weak derivatives of highest order, we are left to show the existence of the intermediate derivatives, which we do in Proposition 22 in the next section. To keep the flow going, we give here the proof that the pointwise inequality implies membership in the corresponding Sobolev space under the assumption that we already know that Proposition 22 holds.

Proof of Theorem 2 (pointwise inequality implies membership in Sobolev space). We combine Lemmas 20 and 21 and apply Proposition 22.

\section{Equivalent Definitions of Sobolev Spaces}

The aim of this section is to prove the following equivalence.

Proposition 22. Let $\Omega \subset \mathbb{R}$ be a domain with finite Lebesgue measure in $\mathbb{R}$. Suppose $X$ is a Banach function space satisfying (B) and (D). Assume that $m \in \mathbb{N}$. Then the following sets coincide:

(a) $A=\{f \in X$ : there exists $F \in X$, where $F$ agrees almost everywhere with $f$ and has weak derivatives up to order $m$ in $X\}$, where the derivatives of order $r, 0 \leq r \leq m-1$, are absolutely continuous,

(b) $B=\left\{f \in X: f^{(k)} \in X, k \in\{0, \ldots, m\}\right\}$, where $f^{(k)}$ are the weak derivatives of $f$, (c) $C=\left\{f \in X: f^{(m)} \in X\right\}$, where $f^{(m)}$ is the weak derivative of $f$ of order $m$.

We see that $A \subset B \subset C$. We now show that $C \subset A$.

Let us give an outline of the proof. Given $g: \Omega \rightarrow \mathbb{R}$ (we will choose $g=f^{(m)}$ ), by integrating, we construct in Lemma 23 an absolutely continuous function $G_{m}$ together with its weak derivatives $G_{0}, \ldots, G_{m-1}$, which are absolutely continuous, and $G_{m}^{(m)}=g$. However, $G_{m}$ and $f$ might not agree almost everywhere. We verify however in the proof of Theorem 26 (with the help of Theorem 24) that we find a polynomial $P$ such that $G_{m}+P$ agrees with $F$ almost everywhere and shares with $G_{m}$ the other required properties. We conclude the proof of Theorem 26 by settling the membership questions with the aid of Proposition 25.

Lemma 23. Assume $\Omega \subset \mathbb{R}$ is a domain. We further assume that $g: \Omega \rightarrow \mathbb{R}$ is in $L_{\text {loc }}^{1}(\Omega)$. One fixes $x^{*} \in \Omega$ and defines

$$
\begin{gathered}
G_{0}\left(x_{0}\right):=g\left(x_{0}\right), \\
G_{k}\left(x_{k}\right):=\int_{x^{*}}^{x_{k}} G_{k-1}\left(x_{k-1}\right) d x_{k-1}, \quad k \in \mathbb{N},
\end{gathered}
$$

where the integrals are Lebesgue integrals, and we use the notation

$$
\begin{aligned}
& \int_{x^{*}}^{x_{k}} G_{k-1}\left(x_{k-1}\right) d x_{k-1} \\
& \quad= \begin{cases}\int_{\left[x^{*}, x_{k}\right]} G_{k-1}\left(x_{k-1}\right) d x_{k-1}, & x^{*} \leq x_{k}, \\
-\int_{\left[x_{k}, x^{*}\right]} G_{k-1}\left(x_{k-1}\right) d x_{k-1}, & x_{k}<x^{*} .\end{cases}
\end{aligned}
$$

Then

(a) $G_{k}$ is locally absolutely continuous for all $k \in \mathbb{N}$, especially locally integrable,

(b) if $\varphi \in \mathscr{C}_{0}^{\infty}(\Omega)$, and $0 \leq r \leq k$, one has

$$
\int_{\Omega} G_{k} \varphi^{(r)}=(-1)^{r} \int_{\Omega} G_{k-r} \varphi .
$$

Proof. Let us prove (a) by induction. Assume $k=1$. Note that $G_{1}\left(x^{*}\right)=0$. Thus, we have for $x^{*} \leq x_{1}$

$$
G_{1}\left(x_{1}\right)-G_{1}\left(x^{*}\right)=G_{1}\left(x_{1}\right)=\int_{x^{*}}^{x_{1}} g\left(x_{0}\right) d x_{0} .
$$

As $g \in L^{1}\left(\left[x^{*}, x_{1}\right]\right)$, we see that $G_{1}$ is absolutely continuous. The case $x_{1}<x^{*}$ is treated similarly.

Assume now that the claim is true for some $k$. Now $G_{k+1}\left(x_{k+1}\right)$ is the integral of a continuous function and thus locally absolutely continuous.

Let us have a look at (b). Note that the statement is true for $k=0$; thus we may assume that $k \geq 1$. We make an induction over $r$. The case $r=0$ is clear. Let us choose an interval $[a, b] \subset \Omega$ containing a neighborhood of the support of $\varphi$. As $G_{k}$ and $\varphi^{(r)}$ are both absolutely continuous in $[a, b]$, we have 
by the integration by part formula for absolutely continuous functions (see, for example, Theorem 7.1.47 in [9]) and the fact that $\varphi^{(r)}(a)=\varphi^{(r)}(b)=0$

$$
\int_{\Omega} G_{k} \varphi^{(r+1)}=\int_{[a, b]} G_{k} \varphi^{(r+1)}=(-1) \int_{[a, b]} G_{k}^{\prime} \varphi^{(r)} .
$$

Noting that $G_{k}^{\prime}=G_{k-1}$ almost everywhere, we see that

$$
\int_{\Omega} G_{k} \varphi^{(r+1)}=(-1) \int_{[a, b]} G_{k-1} \varphi^{(r)}=(-1) \int_{\Omega} G_{k-1} \varphi^{(r)} .
$$

Using the induction hypothesis, the claim follows.

We cite Theorem 2.5.3 in [10] without giving its proof.

Theorem 24. Let $A=[a, b], \mathbb{R}$, or $\mathbb{R}_{+}$and $r \geq 0$. If the function $f \in L_{\mathrm{loc}}^{1}(A)$ satisfies

$$
\int_{A} f \varphi^{(r)} d x=0
$$

for all $\varphi \in \mathscr{C}_{0}^{\infty}(A)$, then there is a polynomial $P$ of degree $<r$ such that $f=P$ almost everywhere on $A$.

To show that $G_{k}$ is in $X$, we need to verify that (if there is danger of confusion with respect to which variable we take the norm, we use notations as $X\left(x_{k}\right)$ )

$$
\left\|\int_{x^{*}}^{x_{k}} G_{k-1}\left(x_{k-1}\right) d x_{k-1}\right\|_{X\left(x_{k}\right)}<\infty
$$

under the assumption that $G_{k-1}$ is in $X$. Actually, bounding $G_{k}$ from above by its absolute value, this means that we have first to take the $L^{1}$-norm and then the norm in $X$. We however would like to switch the norms.

We adapt Proposition 2.1 in [16] to our setting.

Proposition 25. Let $\rho$ be a Banach function norm satisfying property (D). If $f: \Omega \times \Omega \rightarrow \mathbb{R}$ is a measurable function, and one has $f_{x}$ and $f^{y}$ functions on $\Omega$ such that $f_{x}(y)=f(x, y)=$ $f^{y}(x)$, then

$$
\rho\left(\left\|f_{x}\right\|_{1}\right) \leq\left\|\rho\left(f^{y}\right)\right\|_{1} .
$$

The cases $X \in\left\{L^{1}, L^{\infty}\right\}$ are easy to prove. If $X=L^{p}$ for some $1<p<\infty$, then the result can be proven by using Fubini's theorem together with property (D). For the general results we refer to Schep's proof.

The next result builds upon Theorem 2.5.4 in [10].

Theorem 26. Let $\Omega \subset \mathbb{R}$ be a domain, and let $r \geq 1$. Suppose $X$ is a Banach function space satisfying $(B)$ and (D). If $f$ is locally in $X$ and has a generalized $r$ th derivative $g$ locally in $X$, then there is $F$ agreeing almost everywhere with $f$, having absolutely continuous intermediate weak derivatives, and the weak derivative of order $r$ agrees almost everywhere with $g$. Furthermore, the derivatives of $F$ are locally in $X$. If $\Omega$ has finite measure, one obtains the result with "locally" replaced by "globally."
We thank Pilar Silvestre for pointing out how to prove the membership of the intermediate derivatives in $X$.

Proof. Choose $x^{*} \in \Omega$ and let $G_{k}$ be defined as in Lemma 23 . Assume $\varphi \in \mathscr{C}_{0}^{\infty}(\Omega)$. Then, using the fact that $g$ is a weak derivative of $f$ of order $r$ and (b) in Lemma 23

$$
\begin{aligned}
\int_{\Omega}\left(f-G_{r}\right) \varphi^{(r)} d x & =\int_{\Omega} f \varphi^{(r)} d x-\int_{\Omega} G_{r} \varphi^{(r)} d x \\
& =(-1)^{r} \int_{\Omega} g \varphi d x-(-1)^{r} \int_{\Omega} g \varphi d x=0 .
\end{aligned}
$$

Using Theorem 24, we see that $f-G_{r}=P$ almost everywhere, where $P$ is a polynomial of degree $<r$. Thus $f=P+G_{r}$ almost everywhere and for $\varphi \in \mathscr{C}_{0}^{\infty}(\Omega)$ and $0 \leq m \leq r$

$$
\begin{aligned}
\int_{\Omega} f \varphi^{(m)} d x & =\int_{\Omega}\left(P+G_{r}\right) \varphi^{(m)} \\
& =(-1)^{m} \int_{\Omega}\left(G_{r-m}+P^{(m)}\right) \varphi d x .
\end{aligned}
$$

Thus $G_{r-m}+P^{(m)}$ is the $m$ th generalized derivative of $f$. As it is locally absolutely continuous (if $m<r$ ) it lies locally in $X$. In the case $m=r$ the same conclusion holds by the assumption on $g$.

Moreover, there exists $F$, for example, $F=P+G_{r}$, that agrees with $f$ almost everywhere, such that its generalized derivatives up to order $r-1$ are locally absolutely continuous and the $r$ th generalized derivative equals $g$ almost everywhere.

As $g$ is locally in $X$ and the other derivatives are locally absolutely continuous, they are locally in $L^{\infty}$ and thus locally in $X$ by (B).

Suppose now that $\Omega$ has additionally finite measure. Assume that $f$ and $g$ are in $X$ globally. We want to show that the intermediate derivatives are in $X$ as well.

Note in our setting that, by (P5) in Definition 18, membership in $X$ implies membership in $L^{1}$. We proceed by induction. Suppose that $G_{k-1} \in X$. We will apply Proposition 25. We set, denoting by $\operatorname{conv}\left\{x^{*}, x_{k}\right\}$ the closed interval with endpoints $x^{*}$ and $x_{k}$,

$$
f\left(x_{k-1}, x_{k}\right)=G_{k-1}\left(x_{k-1}\right) \chi_{\operatorname{conv}\left\{x^{*}, x_{k}\right\}}\left(x_{k-1}\right) .
$$

Then

$$
\begin{aligned}
& \left\|G_{k}\left(x_{k}\right)\right\|_{X\left(x_{k}\right)} \\
& =\left\|\int_{x^{*}}^{x_{k}} G_{k-1}\left(x_{k-1}\right) d x_{k-1}\right\|_{X\left(x_{k}\right)} \\
& =\left\|\int_{x^{*}}^{x_{k}} f\left(x_{k-1}, x_{k}\right) d x_{k-1}\right\|_{X\left(x_{k}\right)} \\
& \leq\|\| f\left(x_{k-1}, x_{k}\right)\left\|_{L^{1}\left(x_{k-1}\right)}\right\|_{X\left(x_{k}\right)} \\
& \leq\|\| f\left(x_{k-1}, x_{k}\right)\left\|_{X\left(x_{k}\right)}\right\|_{L^{1}\left(x_{k-1}\right)} \\
& \leq\|\| G_{k-1}\left(x_{k-1}\right) \mid\left\|\chi_{\Omega}\right\|_{X\left(x_{k}\right)} \|_{L^{1}\left(x_{k-1}\right)}<\infty .
\end{aligned}
$$




\section{Banach Function Space Setting}

Here, our main focus is a more advanced readership that likely knows already Banach function spaces. Therefore, we adopt a much briefer style, pointing merely to the literature where the corresponding definitions and results can be found. We start with giving generalized versions of the necessity and sufficiency of Theorem 2 .

We list the results and prove them simultaneously by splitting the proofs into parts according to the function spaces.

Theorem 27. Assume $\Omega \subset \mathbb{R}$ is a domain, $X$ is a normed space, and $m \in \mathbb{N}$. If the maximal function $M: X \rightarrow X$ is bounded, then there is a constant $C=C(X, m)$ such that for each continuous $f \in W_{X}^{m}$ there is $a_{f} \in X$ with $\left\|a_{f}\right\| \leq C\|f\|_{W_{X}^{m}}$ and

$$
\left|\Delta^{m} f(x ; y)\right| \leq|x-y|^{m}\left(a_{f}(x)+a_{f}(y)\right) .
$$

In particular such $a_{f}$ exists if

(i) $1<p \leq \infty$ and $X$ is the Lebesgue space $L^{p}(\Omega)$,

(ii) $1<p<\infty, 1 \leq q \leq \infty$, and $X$ is the Lorentz space $L^{p, q}(\Omega)$,

(iii) $1<p<\infty, 1 \leq q \leq \infty, b$ is slowly varying, and $X$ is the Lorentz-Karamata space $L_{p, q ; b}(\Omega)$,

(iv) $\phi$ is an $N$-function with $\inf _{s>0}(s \varphi(s) / \phi(s))>1$, where $\varphi$ is the density function of $\phi$ and $X$ is the Orlicz space $L_{\phi}^{*}(\Omega)$,

(v) $\Omega=\mathbb{R}$ and $X$ is rearrangement-invariant with upper index $\bar{\alpha}_{X}<1$.

Theorem 28. Let $\Omega \subset \mathbb{R}$ be open. Suppose $X$ is a Banach function space such that (AC) holds. Assume further that the function $f: \Omega \rightarrow \mathbb{R}$ is in $X$. If for some $r \in \mathbb{N}_{0}$, the following inequality holds

$$
\left|\Delta^{r} f(x, y)\right| \leq|x-y|^{r}\left[a_{f}(x)+a_{f}(y)\right]
$$

for some $a_{f} \in X$, then there exists $g \in X$ with $\|g\|_{X} \leq 2 r^{r}\left\|a_{f}\right\|_{X}$ such that

$$
\int_{\Omega} f(x) \varphi^{(r)}(x) d x=(-1)^{r} \int_{\Omega} g(x) \varphi(x) d x
$$

for all $\varphi \in \mathscr{C}_{0}^{\infty}(\Omega)$.

In particular the result holds if $X$ is

(i) the Lebesgue space $L^{p}(\Omega)$ for some $1<p<\infty$,

(ii) the Lorentz space $L^{p, q}(\Omega)$, where $1<p<\infty$ and $1 \leq$ $q<\infty$,

(iii) the Lorentz-Karamata space $L_{p, q ; b}(\Omega)$, where $b$ is slowly varying, $1<p<\infty$, and $1<q \leq \infty$, (iv) the Orlicz space $L_{\phi}^{*}(\Omega)$ for some $N$-function $\phi$ satisfying

$$
\inf _{s>0} \frac{s \varphi(s)}{\phi(s)}>1,
$$

where $\varphi$ is the density function of $\phi$.

To help with orientation, let us give an overview of the remaining material in this section. With respect to Theorem 27, note that we carried out the proof of Corollary 14 in a high level of abstraction. Hence, we are left to verify that the spaces under question are normed spaces (we will show that they are even Banach function spaces) and the boundedness of the maximal operator.

Concerning the proof Theorem 28, we proved the corresponding part of Theorem 2 under the assumptions that the spaces under question are Banach function spaces and further satisfy the requirements listed in Properties 19. The existence of $X^{\prime}$ is classical (Proposition 29) as is the fact that (AC) implies (A) and (NC) (Proposition 30). Hence, we only need to point to the literature where $(\mathrm{AC})$ was verified.

Proposition 29. Given a Banach function space, there is a space $X^{\prime}$ such that properties $(B),(H I),(C)$, and $(D)$ are satisfied.

Proof. We refer the reader to Sections 1.1 and 1.2 in [13] or to Section 3.1 in [14].

The definition of absolutely continuous norm can be found as Definition 3.1.11 in [14] and Definition 1.3.1 in [13].

Proposition 30. A Banach function space that has absolutely continuous norm satisfies (A) and (NC).

Proof. The first statement follows from Theorem 1.3.13 in [13] and the second from Proposition 1.3.6 in the same source.

\subsection{General Statements}

Proof of Theorem 27 (general statement). We basically have already proven the general statement in Corollary 14.

Proof of Theorem 28 (general statement). We gave the proof of the Lebesgue space case in such generality that the general statement follows from the proof of Theorem 2 and Propositions 29 and 30.

5.2. Lorentz-Karamata Spaces. Lorentz-Karamata spaces are detailed in [14], mainly Section 3.4.3.

Proof of Theorem 27, (ii) and (iii). The part that is left to prove is the boundedness of the maximal operator, which is guaranteed by Remark 3.5.17 in [14].

Proof of Theorem 28, (ii) and (iii). By Theorem 3.4.41 in [14], we see that $X$ is a rearrangement-invariant Banach space. A combination of Lemma 3.4.39 in [14] together with Lemma 3.4.43 in [14] gives the absolute continuity (AC) of the norm of $X^{\prime}$. 
5.3. Orlicz Spaces. The setting is the one of [17].

Lemma 31. If $\phi$ is an $N$-function satisfying the $\Delta_{2}$-condition, then the Orlicz space $L_{\phi}^{*}$ has absolutely continuous norm.

Proof. Let $f \in L_{\phi}^{*}(\Omega)$. We verify first that

$$
\int_{\Omega} \phi(|f|) d x<\infty
$$

Let $\varepsilon>0$. By the $\Delta_{2}$-condition, there exists a constant $\kappa=$ $\kappa\left(\|f\|_{(\phi)}+\varepsilon\right)$ such that

$$
\phi\left(\left(\|f\|_{(\phi)}+\varepsilon\right) s\right) \leq \kappa \phi(s)
$$

for all $s \geq 0$. Thus

$$
\begin{aligned}
\int_{\Omega} \phi(|f|) d x & =\int_{\Omega} \phi\left(\frac{|f|}{\|f\|_{(\phi)}+\varepsilon}\left(\|f\|_{(\phi)}+\varepsilon\right)\right) d x \\
& \leq \kappa \int_{\Omega} \phi\left(\frac{|f|}{\|f\|_{(\phi)}+\varepsilon}\right) d x \leq \kappa .
\end{aligned}
$$

Suppose that $E$ is a measurable set. Then

$$
\begin{aligned}
\left\|f \chi_{E}\right\|_{(\phi)} & =\inf \left\{\lambda>0: \int_{\Omega} \phi\left(\lambda^{-1}\left|f \chi_{E}\right|\right) d x \leq 1\right\} \\
& =\inf \left\{\lambda>0: \int_{\Omega} \phi\left(\lambda^{-1}|f|\right) \chi_{E} d x \leq 1\right\} .
\end{aligned}
$$

Let us fix $\lambda>0$. Again using the $\Delta_{2}$-condition, we have $\phi\left(\lambda^{-1} s\right) \leq \kappa\left(\lambda^{-1}\right) \phi(s)$. Hence

$$
\int_{\Omega} \phi\left(\lambda^{-1}|f|\right) \chi_{E} d x \leq \kappa \int_{\Omega} \phi(|f|) \chi_{E} d x .
$$

Example 1.3.3 in [13] tells us that $L^{1}$ has absolutely continuous norm. Thus if $\chi_{E_{n}}$ converges $\mu$-almost everywhere to 0 , then $\int_{\Omega} \phi(|f|) \chi_{E_{n}}$ converges to zero as well. Thus, we have for $n$ large enough that

$$
\kappa \int_{\Omega} \phi(|f|) \chi_{E_{n}} d x \leq 1
$$

implying that $\left\|f \chi_{E_{n}}\right\|_{(\phi)} \leq \lambda$. Since $\lambda>0$ was arbitrary, the absolute continuity of $f$ follows.

Proof of Theorem 27 (iv). We conclude from Gallardo's result, Theorem 2.1 in [17], that it is sufficient that the complementary $N$-function satisfies the $\Delta_{2}$-condition in $[0, \infty)$. Again resorting to Gallardo, this time to Proposition 1.4 in [17], we obtain the conclusion.

Proof of Theorem 28 (iv). That $X$ is a Banach function space follows from Theorem 3.4.16 in [14]. To prove that $X^{\prime}$ has absolutely continuous norm, we note that inequality (90) together with Proposition 1.4 in [17] ensures that the complementary $N$-function of $\phi$ satisfies the $\Delta_{2}$ condition. Lemma 31 verifies that $X^{\prime}$ has absolutely continuous norm.
5.4. Rearrangement-Invariant Spaces. The necessary definitions can be found in [13].

Proof of Theorem 27, (v). We are only left to show the boundedness of the maximal operator. However, this is the content of a result by Lorentz and Shimogaki, for example, stated as Theorem 3.5.17 in [13].

\section{Conflict of Interests}

The authors declare that there is no conflict of interests regarding the publication of this paper.

\section{Acknowledgments}

The authors would like to thank Pilar Silvestre for her suggestions of how to prove the existence of intermediate derivatives. The research is supported by the Academy of Finland. The third author thanks the Department of Mathematics and Systems Analysis at Aalto University for its hospitality.

\section{References}

[1] B. Bojarski, "Remarks on some geometric properties of Sobolev mappings," in Functional Analysis \& Related Topics (Sapporo, 1990), pp. 65-76, World Scientific, River Edge, NJ, USA, 1991.

[2] B. Bojarski, P. Hajlasz, and P. Strzelecki, "Improved $C^{k, \lambda}$ approximation of higher order Sobolev functions in norm and capacity," Indiana University Mathematics Journal, vol. 51, no. 3, pp. 507540, 2002.

[3] B. Bojarski, "Pointwise characterization of Sobolev classes," Proceedings of the Steklov Institute of Mathematics, vol. 255, no. 1, pp. 65-81, 2006.

[4] B. Bojarski, "Whitney's jets for Sobolev functions," Ukrainian Mathematical Journal, vol. 59, no. 3, pp. 345-358, 2007.

[5] B. Bojarski, “Taylor expansion and Sobolev spaces," Bulletin of the Georgian National Academy of Sciences. New Series, vol. 5, no. 2, pp. 5-10, 2011.

[6] B. Bojarski, "Sobolev spaces and Lagrange interpolation," Georgian Academy of Sciences. Proceedings of A. Razmadze Mathematical Institute, vol. 158, pp. 1-12, 2012.

[7] P. Hajłasz, "Sobolev spaces on an arbitrary metric space," Potential Analysis, vol. 5, no. 4, pp. 403-415, 1996.

[8] B. Bojarski, "Sobolev spaces and averaging I," http://arxiv.org/ abs/1308.5171.

[9] R. Kannan and C. K. Krueger, Advanced Analysis on the Real Line, Universitext, Springer, New York, NY, USA, 1996.

[10] R. A. DeVore and G. G. Lorentz, Constructive Approximation, vol. 303 of Fundamental Principles of Mathematical Sciences, Springer, Berlin, Germany, 1993.

[11] L. L. Schumaker, Spline Functions: Basic Theory, Cambridge Mathematical Library, Cambridge University Press, Cambridge, UK, 3rd edition, 2007.

[12] G. H. Hardy and J. E. Littlewood, "A maximal theorem with function-theoretic applications," Acta Mathematica, vol. 54, no. 1, pp. 81-116, 1930.

[13] C. Bennett and R. Sharpley, Interpolation of Operators, vol.129 of Pure and Applied Mathematics, Academic Press, Boston, Mass, USA, 1988. 
[14] D. E. Edmunds and W. D. Evans, Hardy Operators, Function Spaces and Embeddings, Springer Monographs in Mathematics, Springer, Berlin, Germany, 2004.

[15] W. Rudin, Real and Complex Analysis, McGraw-Hill Series in Higher Mathematics, McGraw-Hill Book Co., New York, NY, USA, 2nd edition, 1974.

[16] A. R. Schep, "Minkowski's integral inequality for function norms," in Operator Theory in Function Spaces and Banach Lattices, vol. 75 of Operator Theory: Advances and Applications, pp. 299-308, Birkhäuser, Basel, Switzerland, 1995.

[17] D. Gallardo, "Orlicz spaces for which the Hardy-Littlewood maximal operator is bounded," Publicacions Matemàtiques, vol. 32, no. 2, pp. 261-266, 1988. 


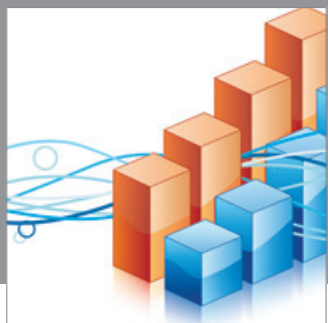

Advances in

Operations Research

mansans

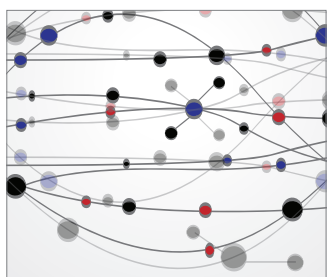

The Scientific World Journal
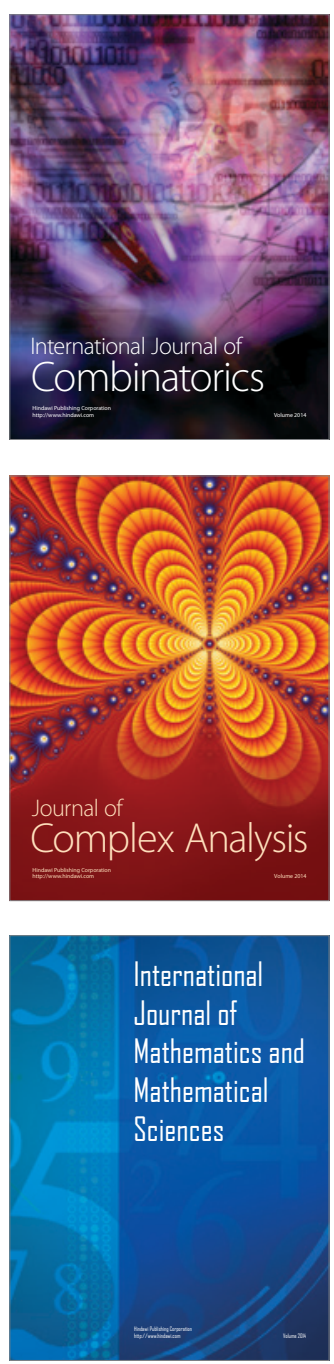
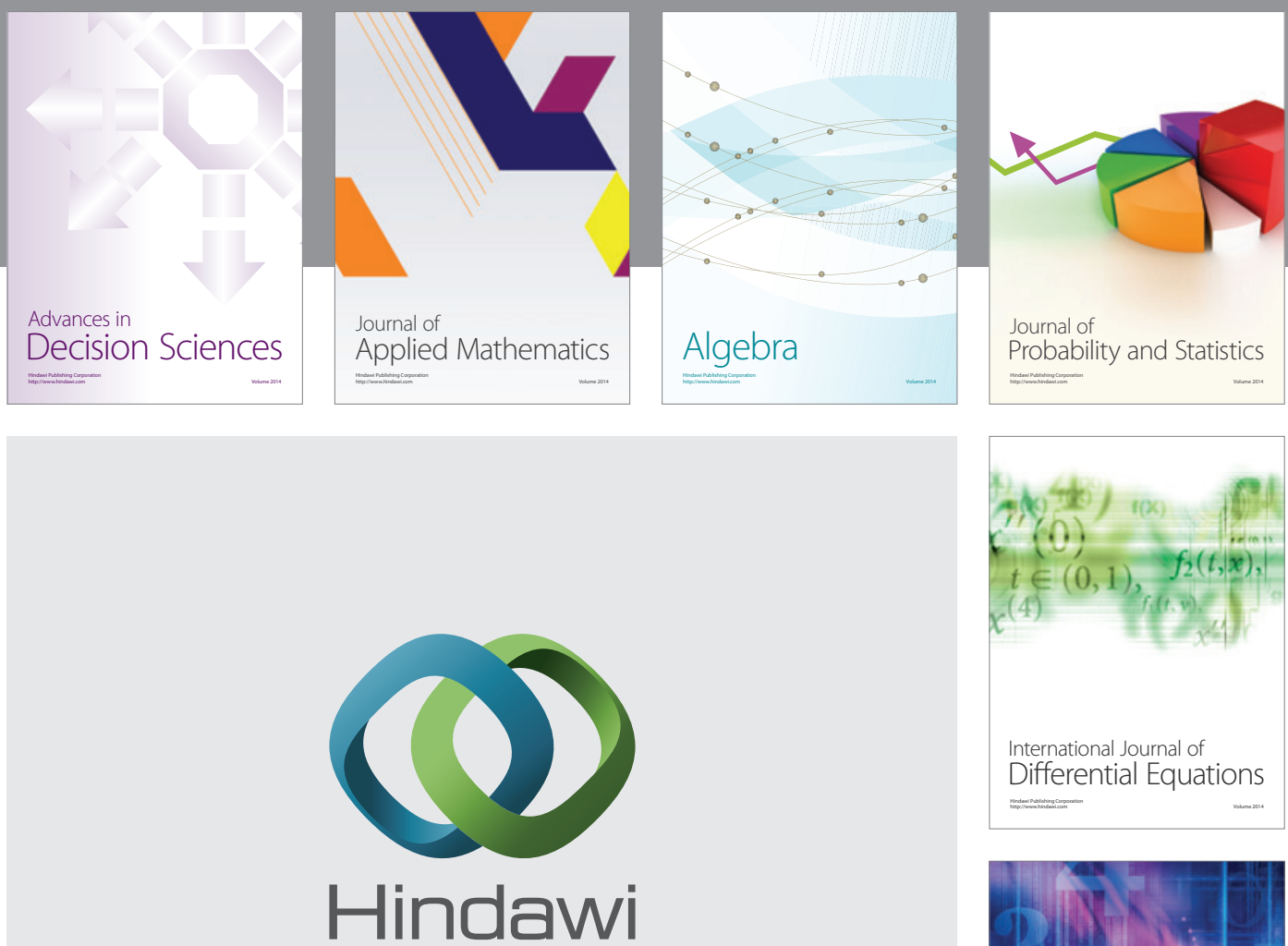

Submit your manuscripts at http://www.hindawi.com
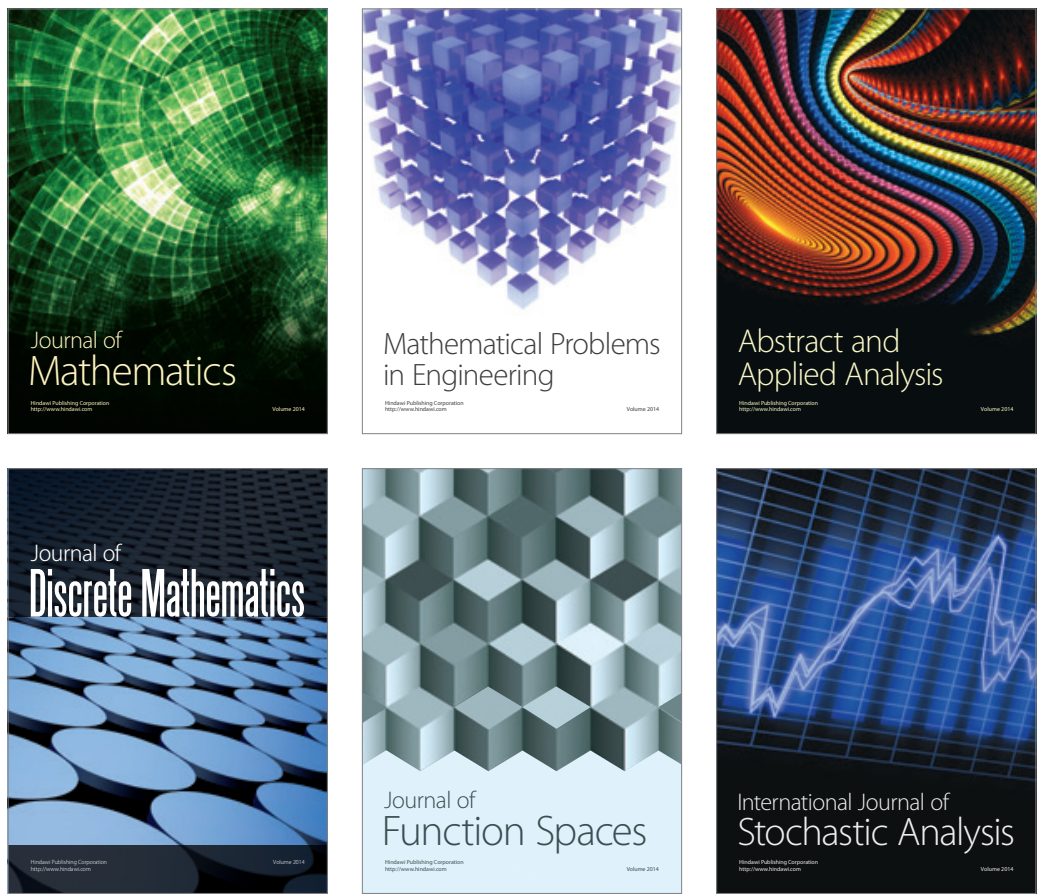

Journal of

Function Spaces

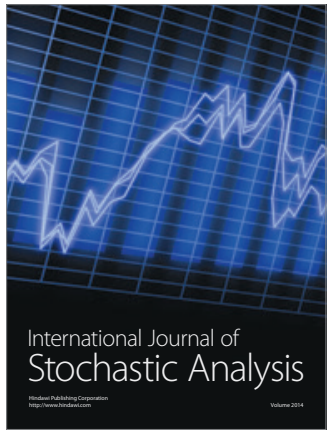

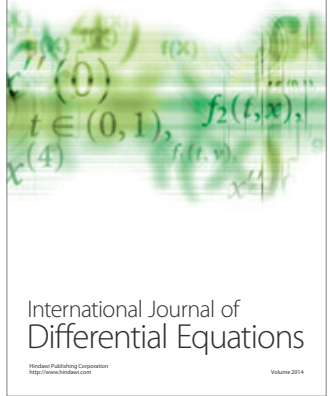
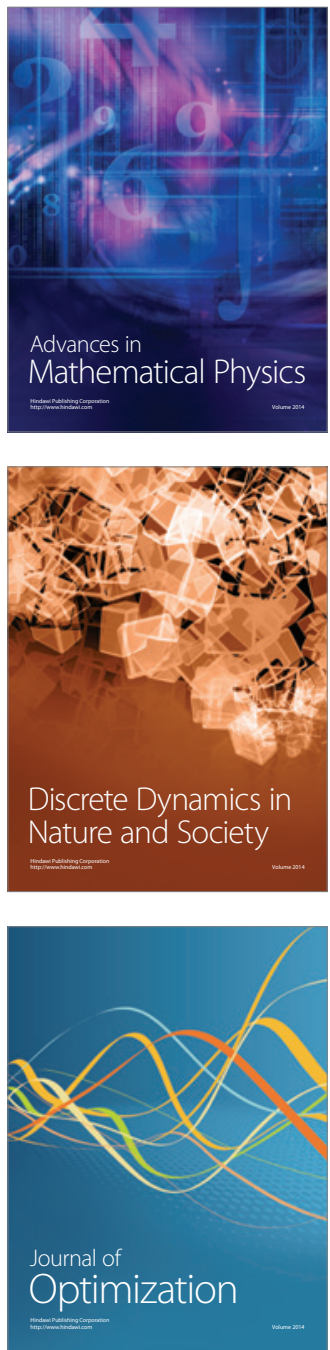